Methods Beginning in the late 1980s, our group in Toronto and Budapest investigated the sensitivity of NTT in over 5000 high-risk gay men, and in an additional 800 men where we could control for HIV status and AIDS. Besides the use of quantified treponemal tests (TT), IgM/IgG screening (in Mardx SDS-PAGE blots), and experimental PCR were employed.

Results The main findings of accelerated screening were undetected and untreated latent cases, not old treated cases as traditionally believed. NTT only detected about 30 percent of cases. Investigators in Houston detected TT IgM in most HIV cases with no NTT reactions, possibly representing latent and chronic cases. Investigators in Vienna showed that aggressive therapy reversed the IgM marker. In Toronto, 557 high risk men were screened with EIA TT and 27 possible latent cases were detected, with negative NTT. 24/27 of these patients had IgM or IgG directed against the main T. pallidum proteins. In the 800 men screened sequentially, TT often dropped in titre or reverted to negative "only" in HIV cases - titres in other routine tests did not drop. Syphilis PCR found that 13/ 183 gay men screened in Hungary had latent syphilis. The PCR found 9 cases negative in both NTT and TT, and identified the four TPHA $(+)$ treated men.

Conclusion NTT is historically unreliable in relapse or reinfection. Syphilis management should always include TT. Syphilis and HIV have an overwhelming association, yet syphilis is never opportunistic. Latent syphilis may be chronically active in many HIV persons. Th- $-\geq$ Th- 2 immunoregulation is the norm in untreated syphilis - many longstanding syphilitics have cutaneous anergy to $\mathrm{TB}$ and mitogens. We suggest a syphilis IGRA be developed.

Disclosure No significant relationships.

\section{P753 MONITORING THE EVALUATION OF CONGENITAL SYPHILIS CASES IN THE STATE OF SÃO PAULO, 2007 TO 2017}

Carla Gianna Luppi, Solange Gomes, Angela Tayra, Mariza Tancredi, Carmen Silvia Domingues*. STI/AIDS Reference Center, Sao Paulo State Program of STI/AIDS, Sao Paulo Department of Health, Sao Paulo, Brazil

10.1136/sextrans-2019-sti.811

Background Congenital syphilis (CS) is still considered a public health problem in the state of São Paulo, Brazil: 4,039 cases were reported in 2017 -incidence rate $=6.6$ per 1,000 live births. The objective of this study was to investigate the evaluation of CS cases in 2007 and 2017 in the state of São Paulo.

Methods A descriptive study was carried out with the data of national surveillance system in the period 2007 to 2017. Of the following evaluation tests performed the percentage of non-performed and positive results were calculated: - Venereal Disease Research Laboratory (VDRL) on the serum; - longbone radiography; -cerebrospinal fluid (CSF) for cell count and protein; - CSF for VDRL.

Results From 2007 to 2017, 21,524 cases of CS born alive were reported: 743 in 2007 and 3,687 in 2017. The percentage of non-performed and positive tests in 2007 and 2017, and the percentage variation (PV) between the two years were: - non-performed - VDRL on the serum $(4.0 \%$ and $1.8 \%$, PV $=-56.3 \%)$, CSF for VDRL $(23.4 \%$ and $21.4 \%$, $\mathrm{PV}=-7.1)$, CSF for cell count and protein $(23 \%$ and
21,4\%: $\mathrm{PV}=7,1 \%)$, long-bone radiography $(13,7 \%$ and $9.9 \%$, PV $=-27.9 \%)$; - positive results - VDRL on the serum $(59.1 \%$ and $81.1 \%$; PV $=37.3 \%)$, CSF for VDRL $(1.3 \%$ and $3.5 \%$; PV $=157.9 \%)$, CSF for cell count and protein $(1.6 \%$ and $10.1 \%$ : PV $=523 \%)$, long-bone radiography $(2.6 \%$ and $3.5 \%, \mathrm{PV}=36.8 \%)$.

Conclusion There was an improvement in adherence to the CS evaluation protocol. In addition, we highlight the increase in the percentage of altered tests of children born alive in the period, pointing to the greater severity of the cases, and the need of improving the monitoring of these children in order to reduce the impact of congenital syphilis.

Disclosure No significant relationships.

\section{P754 QUANTITATION OF CYTOKINES IN RABBITS FOLLOWING TRI-ANTIGEN VACCINE COCKTAIL IMMUNIZATION AND T. PALLIDUM CHALLENGE}

${ }^{1}$ Charmie Godornes*, ${ }^{1}$ Barbara Molini, ' ${ }^{1}$ orenzo Giacani, ${ }^{2}$ Alloysius Gomez, ${ }^{3}$ Darrick Carter ${ }^{2}$ Caroline Cameron, 'Sheila Lukehart. 'University of Washington, Medicine, Seattle, USA; ${ }^{2}$ University of Victoria, Biochemistry and Microbiology, Victoria, Canada; ${ }^{3}$ University of Washington, Global Health, Seattle, USA

\subsection{6/sextrans-2019-sti.812}

Background Immunological analysis of primary and secondary syphilis in rabbits and humans suggests that $\mathrm{T}$ helper cells mount a vigorous interferon- $\gamma$-dominated immune response $\left(\mathrm{T}_{\mathrm{H}} 1\right)$ to facilitate macrophage-mediated clearance of $T$. pallidum. In this study, we used quantitative reverse transcriptase (qRT)-PCR to evaluate post-challenge rabbit cytokine profiles of primary lesions in unimmunized rabbits and rabbits immunized with a tri-antigen vaccine cocktail.

Methods Groups of 8 male New Zealand White rabbits were immunized with a trivalent recombinant antigen cocktail, $(\mathrm{N}$ term of $\mathrm{TprK}+\mathrm{N}$ - term of Tpr Subfamily I $+\mathrm{Tp} 0751$, emulsified in either of two custom adjuvants containing Natural or Synthetic TLR4 agonists + a natural Mincle agonist). Unimmunized control and immunized animals were intradermally challenged with $10^{5} \mathrm{~T}$. pallidum (Nichols) at each of 10 sites. Lesion biopsies were collected at days 2 and 21 postchallenge. Expression of IFN- $\gamma$, TGF- $\beta$, p40 IL-12/23, IL-4, IL-2, TNF- $\alpha$, IL-10, IL-17A, IL-17F, and IL-22 was quantified by qRT-PCR using plasmids containing the target rabbit cytokines sequences, and expression levels were normalized to rabbit HPRT.

Results At day 2, transcripts for IFN- $\gamma$, IL-2, IL-17A, IL-17F, and TNF- $\alpha$ were significantly upregulated in both immunized groups $(\mathrm{P}<0.01)$ compared to controls, a finding consistent with the development of clinical delayed type hypersensitivity and the induction of a $\mathrm{T}_{\mathrm{H}}$ 1-type immune response at challenge sites. At day 21, the level of IFN- $\gamma$ was lower in the Natural adjuvant group $(\mathrm{P}<0.02)$, compared to controls, consistent with enhanced treponemal clearance in that group.

Conclusion In the development of a syphilis vaccine, it is important to determine correlates of protection to allow for assessment of the induction of a protective immune response. Our results demonstrate a robust immunization-induced $\mathrm{T}_{\mathrm{H}} 1$ and $\mathrm{T}_{\mathrm{H}} 17$ proinflammatory response in immunized rabbit groups, which suggests an effective level of resistance conferred by immunization with the trivalent protein vaccine. Disclosure No significant relationships. 